\title{
Studies of Roughness Induced Transition at Mach 6 Using IR Thermography
}

\author{
by C. O. Asma, A. Garzón, S. Paris and D. Fletcher
}

von Karman Institute, Rhode Saint Genèse, Belgium

\begin{abstract}
Transition on the boundary layer of a hypersonic vehicle can result from many factors, among which one of the most important is the presence of discontinuities (roughness) on its surface. For experiments performed in ground facilities, not all the in-flight conditions can be reproduced resulting in a mismatch between the data obtained from experimentation and the real flight conditions. This work represents an initial investigation of the parameters for transition in the Mach $6 \mathrm{H} 3 \mathrm{VKI}$ wind tunnel using infrared thermography and on Kheops model. Three different kinds of discontinuities are considered: distributed roughness, gaps between different sections and isolated roughness elements. Results from the $\mathrm{H} 3$ tests are found to be in good agreement with ballistics range results plotted with PANT criterion parameters.
\end{abstract}

\section{Introduction}

Aerodynamic heating during an atmospheric re-entry can be drastically increased when the boundary layer transitions from laminar to turbulent. This transition of the boundary layer nature can be caused by a wide range of factors, among which one of the most important is the presence of discontinuities on the surface along which the boundary layer is developed. The understanding of transition in hypersonic flows is of great importance since it can help in designing more efficient vehicles.

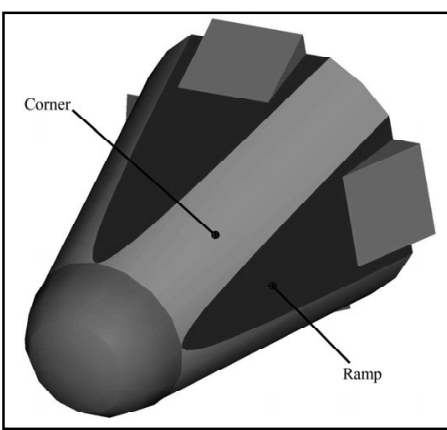

Fig. 1. Kheops Model

The EXPERT project is an initiative of the European Space Agency that aims to improve the knowledge about the gap between the experiments and real conditions of flight through an in-flight experience [1]. The program is based on the ballistic reentry trajectory of the Kheops model (see Fig. 1), which is installed inside the head of a VOLNA missile. Among the different payloads are experiments designed to measure boundary layer transition from laminar to turbulent flow. One of the main goals of the EXPERT project is to follow the development of the boundary layer at real re-entry conditions and analyze the possible triggering mechanisms to such a state. This work is intended to investigate candidate distributed and isolated roughness configurations to evaluate their effectiveness in inducing transition for a Kheops flight configuration.

\section{Experimental Facility and Instrumentation}

\subsection{The $\mathrm{H} 3$ Wind Tunnel}

The H3 is a hypersonic blow-down wind tunnel. It has an axisymmetric nozzle that provides a Mach 6 flow. Air is supplied from a pebble-bed heater at stagnation 
pressures ranging from 7 to 35 bar and maximum stagnation temperature of $550 \mathrm{~K}$. The unit free-stream Reynolds number can be varied from $3 \times 10^{6} \mathrm{~m}^{-1}$ to $3 \times 10^{7} \mathrm{~m}^{-1}$. The model can be injected into the flow by means of a three-degree of freedom traversing system. The mechanism also allows a rapid injection. The flow field is considered to be uniform. The variation of the Mach number at a distance of $100 \mathrm{~mm}$ from the nozzle is found to be between 5.92 and 6.07 on [2]. The stagnation temperature and pressure of the wind tunnel are measured with a calibrated chromel-alumel thermocouple and a Statham (strain-gauge) pressure transducer. The uncertainties on the measurements are $\delta \mathrm{P}_{0}= \pm 0.25 \%(1: 20)$ and $\delta \mathrm{T}_{0}= \pm 1 \%(1: 20)$, resulting in an overall uncertainty of $\pm 6.29 \%$ (1:40) for Reynolds number. [3].

\subsection{The Infrared Camera}

An AGEMA 900 infrared camera and image recording system are used to measure the evolution of temperature in time on the walls of the model. The infrared camera intensities are converted to temperatures using calibrated thermocouples and a black painted flat plate heated to a desired temperature. The overall uncertainty of the wall temperature measurements is calculated to be $\delta \mathrm{T}_{\mathrm{w}} / \mathrm{T}_{\mathrm{w}}= \pm 2.7 \mathrm{~K}$ $(1: 10)$ [3], resulting in a final uncertainty of $\pm 10 \%$ in the Stanton number values [4].

\subsection{The Model}

The KHEOPS configuration 4.1 is used as the test model. The shape is a body of revolution with an ellipse-clothoid-cone profile with a total length of $1.6 \mathrm{~m}$. The angle of the cone is $12.5^{\circ}$; four planes of $9^{\circ}$ inclinations intersect the body generating four ramps of $9^{\circ}$ with respect to a center-line. This ramps start at the end of the clothoid-cone junction line. At the end of the ramps a flap of $20^{\circ}$ with respect to the ramp is set (see Fig. 1). A scaled model with a total length of $109 \mathrm{~mm}$ is used during the experiments.

\subsubsection{Exchangeable Roughness Elements}

Since the goal of the project is to determine the location where transition is likely to happen, owing to the presence of disturbing elements, a special region was designed to support them. Several configurations with distributed roughness, gaps, and isolated roughness elements were considered. Rings with different roughness elements are manufactured to be installed at the light-grey region shown in Fig. 2.

For distributed roughness, two different sand papers are used: 1) commercial P60 sand paper (S1) and 2) a kind of anti-slip strip (S2) placed on stair steps. When attached to the surface both materials did protrude about half their thickness to have an efficient disturbance height. Another distributed roughness was made by using a lathe to apply a knurl (K1) to the original piece, creating a diamond pattern. Last of the distributed roughness

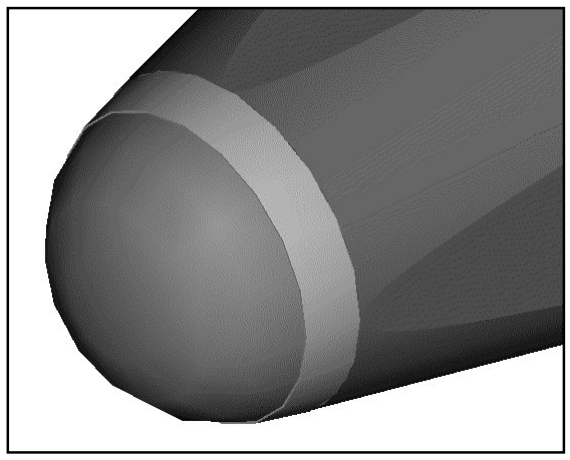

Fig. 2. Nose-body junction elements is a "2D" tripping wire element (T1), which is mounted along the dip element (see below paragraph).

For the gaps, six different cases are considered. The first one (D1) is a $1 \times 1 \mathrm{~mm}$ dip in the middle of the ring piece. D2, D3 and D4 are gaps generated by inserting a few spheres of $2 \mathrm{~mm}$ and $1 \mathrm{~mm}$ of diameter between the ring and the body. For the 
cases D5 and D6, spheres of $2 \mathrm{~mm}$ and $1 \mathrm{~mm}$ have been put in between the nose and the roughness ring.

For the isolated roughness elements, several different cylindrical-roughnesselement configurations are considered. A single geometry was designed, with the characteristic of allowing the adjustment in the roughness height. The cylinders used are $2 \mathrm{~mm}$ in diameter. Figure 3 presents photos of different roughness elements installed on the model.

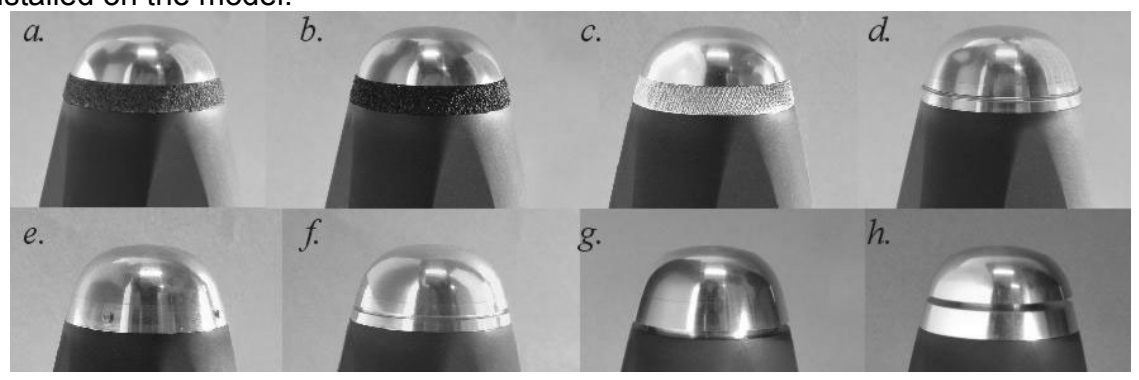

Fig. 3. Different roughness configurations:

a. Sand Paper P60 b. Anti slip paper c. Knurled d. Tripping wire e. Isolated cylinders $\boldsymbol{f}$. Dip $1 \mathrm{~mm} \boldsymbol{g}$. Gap between body and ring $\boldsymbol{h}$. Gap between nose and ring

\section{Experimental Results}

The model is tested in $\mathrm{H} 3$ wind tunnel for smooth and all roughness configurations at 10 and 30 bar stagnation pressure conditions. Repeatability tests are performed for some cases, and these exhibitedgood repeatability. The surface temperature both on the corner and on the ramp sections of the model (see Fig. 1) is recorded for a minimum duration of 2 seconds using the AGEMA 900 infrared camera with a frequency of $15 \mathrm{~Hz}$. The time-history of temperature on the centerline of the model is processed to get the heat flux and thus the modified Stanton number, which is the non-dimensional heat flux. Investigation of temperature contours and Stanton number distribution gives an idea of whether the flow is laminar or turbulent on the model.

$$
S t=\frac{\dot{q}_{w}}{C_{p} \rho_{\infty} u_{\infty}\left(T_{a w}-T_{w}\right)}
$$

\subsection{Distributed Roughness Results}

The normalized plots for both ramp and corner cases can be seen in Fig. 4a 4.b, respectively. The $\mathrm{x}$-axis is presented in terms of $S t \cdot \sqrt{\operatorname{Re}_{x}}$ to be able to differentiate laminar and turbulent cases. Turbulent cases are seen to group at a high level of $S t \cdot \sqrt{\operatorname{Re}_{x}}$ while laminar values occupy the low region of the plot. A clearly transitional case is also observed for the tripping wire test case at 30 bar for both the ramp and the corner zone. Transitional and fully turbulent boundary layers are found only for the 30 bar test cases. Even though there is not a collapse of all the turbulent results as there is collapse for the laminar cases, a clear differentiation of the two regimes is observed. 

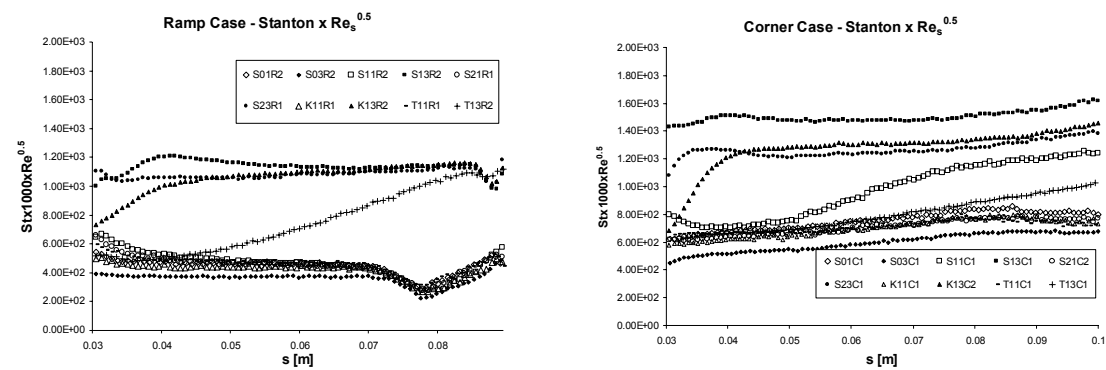

Fig. 4. Distributed roughness: $S t \cdot \sqrt{\mathrm{Re}_{x}}$ distribution for a) ramp b) corner

\subsection{Dip and Gap Results}

Based on the same criterion applied for the test cases on the distributed roughness elements, results for the dip and the different gaps have also been plotted under the same Reynolds number scaling on Fig. $5 a$ and $5 b$, for the ramp and corner respectively. The same vertical scale is maintained with respect to the distributed roughness plots and, as a reference, a laminar (smooth), turbulent (anti-slip paper) and transitional (tripping wire) test cases are included.
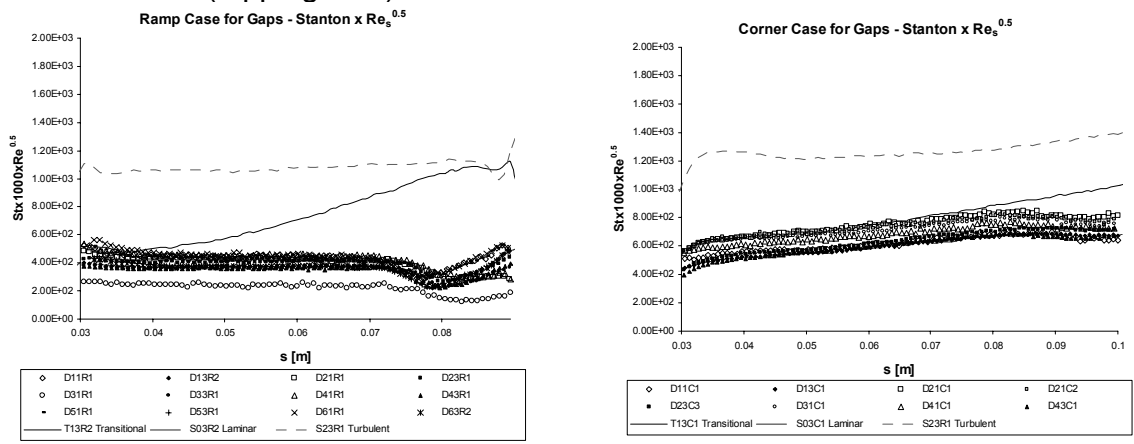

Fig. 5. Dip and gap results: $S t \cdot \sqrt{\mathrm{Re}_{x}}$ distribution for a) ramp b) corner

\subsection{Isolated Roughness Element Results}

For the testing of isolated cylindrical-roughness elements, the tests were performed only on the corner since it is on this point of the vehicle that transition experiments will be installed on the Kheops vehicle. The Stanton number plotting for this kind of roughness had to be done differently, since the Stanton number changes dramatically from line to line due to the presence of detached vortices and the transverse expansion of the wake. A different approach is thus used and contours for both temperature and Stanton number are drawn. To obtain the Stanton number contour it is first necessary to define what is the zone of interest, so temperature contours are first observed. The results for temperature contours 30 bar test cases are shown in Fig. 6. The respective Stanton number pictures are shown in Fig. 7. 


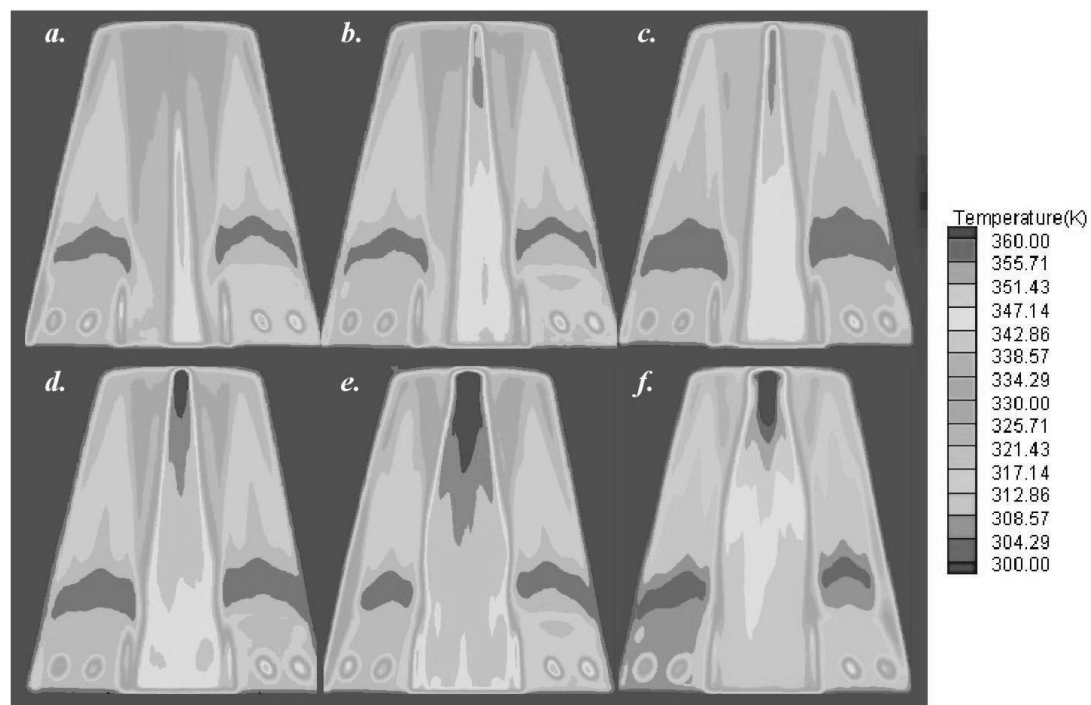

Fig. 6. Temperature contours at the wall for isolated roughness element $(2 \mathrm{~mm} \varnothing$ cylinder); 30 bar at 1.666 seconds after injection. a. $k=100 \mu \mathrm{m}$ b. $k=200 \mu \mathrm{m} \mathrm{c}$.

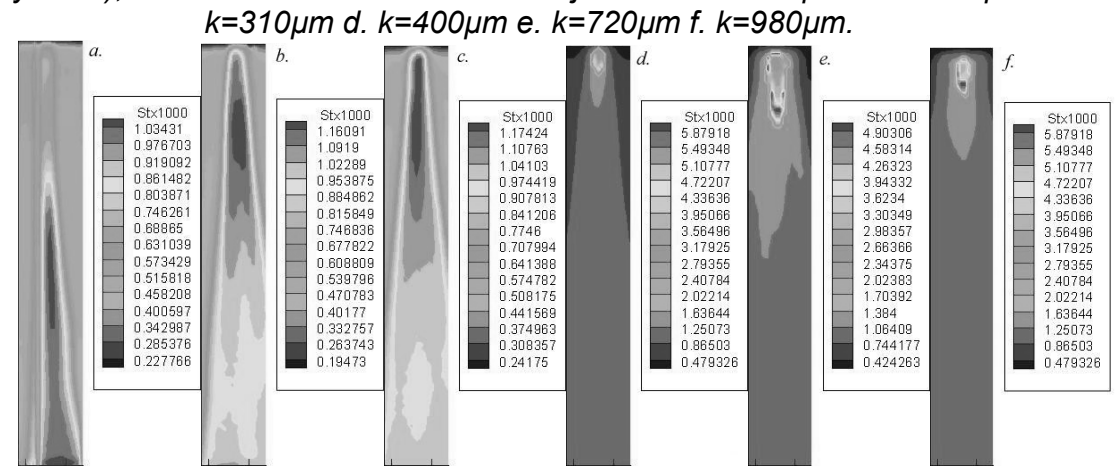

Fig. 7 Stanton number contours at the wall for isolated roughness element (2mm $\varnothing$ cylinder); 30 bar at 1.666 seconds after injection. a. $k=100 \mu \mathrm{m} \mathrm{b.} k=200 \mu \mathrm{m} \mathrm{c}$. $k=310 \mu \mathrm{m}$ d. $k=400 \mu \mathrm{m}$ e. $k=720 \mu \mathrm{m}$. $k=980 \mu \mathrm{m}$.

\section{Discussion and Conclusion}

Considering that there is no analytical means to predict transition, and since relationships between physical properties and transition are not clearly known; empirical correlations are currently used to compare experimental results. When experimental results based on transition are found it becomes necessary to be able to compare them, and a transition correlation is thus necessary. Two parameters that represent the flow conditions at which transition occurs are usually chosen. The first parameter is called the transition parameter, and traditionally includes the Reynolds number based on the boundary layer edge conditions at the location of the perturbation and the momentum thickness as the characteristic length. The second parameter is called the disturbance parameter and usually involves a relation between a characteristic height of a roughness element and some boundary layer characteristic dimension such as boundary layer thickness, momentum thickness or 


\section{http://dx.doi.org/10.21611/qirt.2004.086}

even displacement thickness. Below is a comparison and discussion of the experimental results with some existing transition criteria.

\subsection{The Shuttle Criterion}

The Shuttle criterion uses as a transition parameter the Reynolds number and the Mach number based on the edge conditions of the boundary layer and the momentum thickness of the boundary layer at the point where the disturbance parameter is located. The disturbance parameter is the simplest one, the ratio of the roughness characteristic height to the boundary layer thickness. This criterion was developed mainly to account for the effect of isolated roughness elements.

$$
\frac{R e_{\theta}}{M_{e}}=C\left(\frac{k}{\delta}\right)^{-1}
$$

The constant $C$ delimits the lam-trans and trans-turb zones defined by the correlation and is found by fitting the plotted results [5]. The results with isolated roughness elements are plotted in Fig. 8 and compared with Space Shuttle fits $(C=21$ and $C=30$ for effective and incipient transition). It can be seen that transitional flow is found at values of lower $C$ than those for the Space Shuttle.

\subsection{The PANT Criterion}

The PANT type criteria for transition is characterized by the representation of both the disturbance and transition parameters plotted on a log-log graph. The empirical correlation obtained from PANT criteria initially by A.D. Anderson in 1975 uses $R_{e}$ as a transition parameter and the ratio between the products of roughness height and temperature at the edge of the boundary layer with momentum thickness and wall temperature as a disturbance parameter. The relation is as follows [6]:

$$
R e_{\theta, T R}=215\left(\frac{k}{\theta} \frac{T_{e}}{T_{w}}\right)^{-0.7}
$$

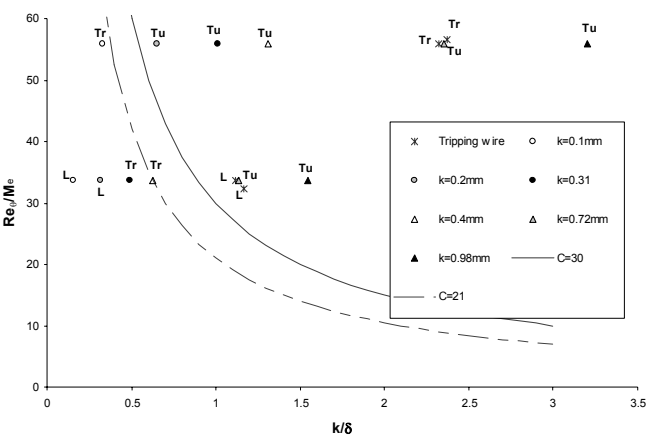

Fig. 8. Shuttle criterion for isolated roughness

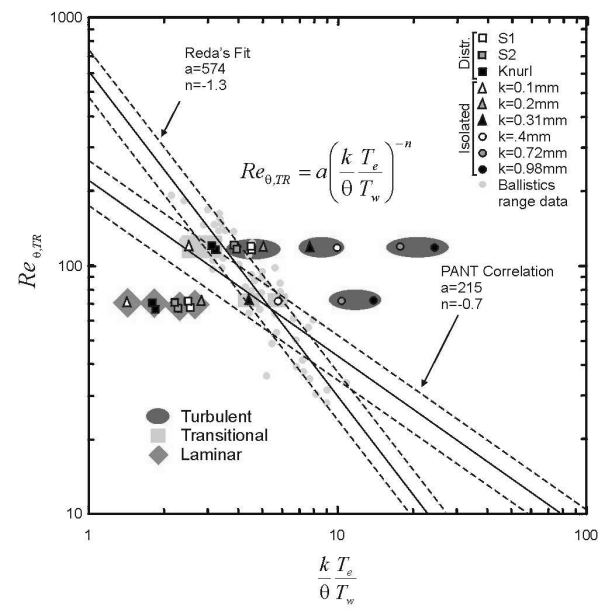

Fig. 9. PANT and Reda criteria

A second database was obtained by Reda [7] who reported a significant number of tests in a ballistics range. Reda's correlation was found to be in good agreement with Anderson's, though he suggests the use of different coefficients which fit better with his data base: 574 for the constant and -1.3 for the exponent.

The results for turbulent, laminar and transitional cases, including isolated roughness are shown in Fig. 9. for the sake of comparison. The higher line of results corresponds to the high-pressure tests (high Reynolds number) values. It is interesting to see the good agreement that the results for isolated roughness 
elements present with an experimental criterion for distributed roughness. It is important to remember that such agreement is not seen when the results are plotted through Shuttle criterion. This puts in evidence the stronger physical background that PANT correlation has when compared to a criterion like Shuttle, giving it a more universal character.

\section{REFERENCES}

[1] FESART/EXPERT Aerodynamic and Aerothermodynamic analysis of the REV and KHEOPS configurations. Technical Report ESA-ESTEC September 2002.

[2] Boerrigter, H. L. Calibration of the $\mathrm{H} 3$ wind tunnel using Pitot probes. Internal Note 94. von Karman Institute, Rhode Saint Genèse, Belgium, December 1993.

[3] Garzon, A., Roughness Induced Transition for Flight Experiments. PR 2003-13. von Karman Institute, Rhode Saint Genèse, Belgium, June 2003.

[4] C. O. Asma. Transition and shock wave interaction in hypersonic flows. PR 2001-01, von Karman Institute, Rhode Saint Genèse, Belgium, June 2001.

[5] Berry, S.A., Bouslog, S. A., Brauckmann G. J. \& Caram, J. M.: Shuttler Orbiter experimental boundary layer transition results with isolated roughness. J. Spacecraft and Rockets, Vol. 35, No 3, May-June 1998, pp 241-248

[6] Anderson., J. D.: Hypersonic and High Temperature Gas Dynamics. AIAA. 2000.

[7] Reda, D. C.: Corrrelation of nosetip boundary layer transition data measured in ballistics-range experiments. AIAA Journal, Vol. 19, No 3, March 1981, pp 329339. 
http://dx.doi.org/10.21611/qirt.2004.086 